\title{
Evaluation of screening tests for autoimmune gastritis in histopathologically confirmed Japanese patients, and re-evaluation of histopathological classification
}

\section{Yasuhiro Wada ( $\square$ wadayasuhiro@oita-u.ac.jp )}

Oita University: Oita Daigaku

\section{Shigemi Nakajima}

JCHO Shiga Hospital; Shiga University of Medical Science https://orcid.org/0000-0003-1386-0985

Naoko Mori

JCHO Shiga Hospital; Shiga University of Medical Science: Shiga Ika Daigaku

\section{Shizuki Takemura}

Kusatsu General Hospital: Kusatsu Sogo Byoin

\section{Rena Chatani}

JCHO Shiga Hospital; Shiga University of Medical Science

\section{Mariko Ohara}

JCHO Shiga Hospital; Shiga University of Medical Science

\section{Makoto Fujii}

JCHO Shiga Hospital; Shiga University of Medical Science

\section{Hiroshi Hasegawa}

JCHO Shiga Hospital; Shiga University of Medical Science

\section{Kiyoyuki Hayafuji}

JCHO Shiga Hospital; Shiga University of Medical Science

\section{Ryoji Kushima}

Shiga University of Medical Science: Shiga Ika Daigaku

\section{Kazunari Murakami}

Oita University Faculty Of Medicine Graduate School of Medicine: Oita Daigaku Igakubu Daigakuin Igakukei Kenkyuka

\section{Research Article}

Keywords: anti-parietal cell antibody, gastrin, corpus atrophy, pepsinogen, vitamin B12

Posted Date: March 1st, 2022 
DOI: https://doi.org/10.21203/rs.3.rs-1210431/v1

License: (c) (i) This work is licensed under a Creative Commons Attribution 4.0 International License. Read Full License

Version of Record: A version of this preprint was published at BMC Gastroenterology on April 11th, 2022. See the published version at https://doi.org/10.1186/s12876-022-02251-8. 


\section{Abstract}

Background. The aims of the present study are to evaluate non-invasive screening tests for autoimmune gastritis (AIG) and re-evaluate histopathological classification.

Methods. We screened candidates of AIG in JCHO Shiga Hospital between May 2012 and January 2020. The screening criteria were as follows: endoscopic 0-p atrophy with Updated Kimura-Takemoto classification, $3+$ pepsinogen $(P G)$ test, low serum vitamin $B_{12}$ or elevated serum gastrin with positive anti-parietal cell (PC) or intrinsic factor antibodies. We evaluated the screening criteria in the patients who were histopathologically confirmed as AIG, and re-evaluated histopathological staging in clinical aspects.

Results. Twenty-two of 28 (78.6\%) patients who met the screening criteria were histopathologically confirmed as AIG. Common clinical findings in the AIG patients were 10x or greater anti-PC antibody, elevated serum gastrin greater than $172 \mathrm{pg} / \mathrm{mL}$ and endoscopic atrophy 0-1 or greater. The areas under the curve of PG I, PG II and PG I/II ratio were $0.81,0.29$ and 0.98 , respectively. Among histopathologically confirmed AIG patients, 4 and 18 patients were histopathologically classified into florid and end stages, respectively, while no patients into early stage. We could not find a significant difference between florid and end stages in the screening items studied.

Conclusions. Florid and end stages in histopathological classification are both advanced-stage AIG in clinical aspects. Our screening criteria without biopsy are applicable to screen clinically-advanced AIG with $78.6 \%$ positive predictive value. PG I and PG I/II ratio may be useful to screen AIG. However, we may need other criteria to screen early stage of AIG.

\section{Background}

Autoimmune gastritis (AIG) was summarized in 1973 by Strickland and Mackey as diseases with fundic gland atrophy, decreased gastric acid secretion, positive anti-parietal cell (PC) antibody or anti-intrinsic factor (IF) antibody and elevated serum gastrin often accompanied with pernicious anemia [1]. AIG had been considered uncommon in Asian countries until recently [2,3]. However, some studies in Japan reported that the actual prevalence of AIG would be higher than believed. Notsu et al. reported that the prevalence of AIG was at least $0.49 \%$ in 6739 subjects with endoscopic upper GI screening [4]. On the other hand, much more undiagnosed candidates of AIG were found in Western countries $[5,6]$. In Germany, anti-PC antibody is present in 19.5\% of the 9684 individuals undergone health checkups [6]. To know the precise prevalence of AIG, we need large number of subjects to be screened.

Currently, the final diagnosis of AIG often depends on histopathological findings in biopsy specimens which are taken with an invasive procedure. Before taking biopsy specimens we need clinical criteria to screen AIG without invasive procedures. Serum gastrin may be a good tool to screen AIG, but we do not know whether it is enough. To screen AIG, fundic gland atrophy is essential. Endoscopic evaluation of atrophy is established by Kimura and Takemoto in 1968, and recently it has been updated [7, 8]. Serum pepsinogen (PG) test have been used as biomarkers of gastric atrophy and cancer risk [9]. Thus 
endoscopic evaluation of gastric atrophy and PG test may be useful to screen AIG. In addition, serum vitamin $B_{12}$ may be a good tool to screen IF deficiency in AIG. We have recently proposed screening criteria of AIG including these tests to screen more patients with AIG [10], so we have to clarify whether the criteria are appropriate in histopathologically confirmed AIG. Histopathologically, AIG have been classified into early, florid and end stages [11-13], but the clinical differences between these stages are not clearly elucidated.

In the present study, we evaluated our screening criteria for AIG in histopathological aspects and reevaluated the histopathological classification in clinical aspects.

\section{Methods}

\section{Subjects}

We collected medical information of the candidates who were clinically suspected of AIG in Japan Community Healthcare Organization (JCHO) Shiga Hospital between May 2012 and January 2020. The screening criteria for candidates were as in the followings: marked endoscopic corpus atrophy (0-p in Updated Kimura-Takemoto classification) [7], severely positive (3+) PG test, low serum vitamin $B_{12}$ or elevated serum gastrin, accompanied with positive anti-PC or IF antibodies[10]. Among them, those who were taken biopsy specimens were histopathologically evaluated as described below.

\section{Tests and the Procedures}

Anti-PC antibody and anti-IF antibody were measured with anti-mitochondrial antibody/anti-smooth muscle antibody FA (Fujirebio, Tokyo, Japan) and Beckman Coulter ACCESS intrinsic factor Ab (Beckman Coulter, Brea, USA), respectively. Because the above tests were not covered with medical insurance in Japan, we could not examine all the suspected patients. If the patient accepted to pay, we tested anti-PC antibody first, and then tested anti-IF antibody if the first one was negative and the patient accepted to pay for the next one. Those who did not accept the tests were not included in the candidates. Endoscopic gastric atrophy was evaluated with Updated Kimura-Takemoto classification [7, 8]. Briefly endoscopic atrophic border was classified into 8 grades: C-0, no atrophy; C-1, closed border in the antrum; C-2, closed border in the distal corpus; $\mathrm{C}-3$, closed border in the proximal corpus; 0-1, open border in the lessor curvature; 0-2, open border between lessor and greater curvatures; 0-3, open border in the greater curvature; 0-p, pan atrophy in the corpus without atrophic border. Serum PG was measured with ARCHITECT Pepsinogen I and II (Abbott Japan, Tokyo, Japan). According to Miki and Urita [9], all patients were classified into four groups: severely positive (3+), serum PG I $\leq 30 \mathrm{ng} / \mathrm{ml}$ and PG I/II ratio $\leq 2.0$; moderately positive (2+), serum PG I $\leq 50 \mathrm{ng} / \mathrm{ml}$ and $P G \mathrm{I} / \mathrm{II}$ ratio $\leq 3.0$; mildly positive $(+)$, serum $P G$ I $\leq 70 \mathrm{ng} / \mathrm{ml}$ and PG I/II ratio $\leq 3.0$; and negative (-), the remainings. Because PG tests were not covered by medical insurance in Japan, we did not test them in all the candidates. Serum gastrin and vitamin $B_{12}$ were measured with Gastrin RIA Kit II (Fujirebio) and Beckman Coulter ACCESS B 12 (Beckman Coulter), 
respectively. Values higher than $172 \mathrm{pg} / \mathrm{mL}$ for gastrin or values lower than $180 \mathrm{pg} / \mathrm{mL}$ for vitamin $\mathrm{B}_{12}$ were defined high or low, respectively, according to the manufacturer's instruction.

\section{Histopathological diagnosis}

For histopathological evaluation, biopsy specimens were endoscopically taken from the greater curvature of the middle corpus and the antrum. Biopsy specimens were immediately fixed in $10 \%$ neutral buffered formalin for $24 \mathrm{~h}$ and embedded in paraffin. Samples were sliced into $3 \mu \mathrm{m}$-thick sections and stained with hematoxylin and eosin (H\&E). We histopathologically confirmed AIG by observing the status of inflammatory cell infiltration and atrophy of lamina propria in the middle corpus mucosa, while comparing the findings of antral mucosa. AIG was divided into three stages. The early stage consisted of patchily decreased fundic glands with diffuse or multifocal dense basal-predominant lymphoplasmacytic infiltration. The florid stage consisted of marked decrease of fundic glands with lymphoplasmacytic infiltration. The end stage consisted of complete parietal cell loss with minimal inflammatory cell infiltration [11-13]. Atrophy of fundic glands included marked decrease or loss of fundic glands with or without pyloric or intestinal metaplasia. Enterochromaffin cell-like (ECL) cell hyperplasia in the corpus and gastrin cell hyperplasia in the antrum were supporting findings to diagnose AIG. Histopathological diagnosis was performed by two pathologists and consensus was made if the diagnoses were different.

Helicobacter pylori (H. pylori) infection

In order to confirm the infection status of $H$. pylori, history about the past $H$. pylori tests and therapies was collected from the medical records. H. pylori tests included serum H. pylori antibody test (HpAb) (Eplate Eiken H. pylori antibody II, Eiken Kagaku, Tochigi, Japan), H. pylori stool antigen test (HpSA) (Meridian HpSA ELISA II, Fujirebio), urea breath test (UBT) (Ubit Tablets 100 mg, Otsuka Pharmaceutical, Tokyo, Japan; POCone, Otsuka Electronics, Osaka, Japan), histopathology (H\&E staining and Giemsa staining) and culture for $H$. pylori. Serum anti-H. pylori was tentatively judged negative when the ELISA value was below $10.0 \mathrm{U} / \mathrm{mL}$ according to manufacturer's instruction. HpSA was tentatively judged positive with the value 0.120 or more and negative with the values less than 0.100 . UBT was tentatively judged negative with the $\Delta^{13} \mathrm{CO} 2$ (UBT value) less than $2.0 \%$ ond positive with the value $5.0 \%$ or more. Patients with clear evidence of current $H$. pylori infection with pathology or culture were determined "currently infected". Those showing no evidence of infection by all the tests performed were diagnosed "not infected". Those who had past eradication therapy were included in "past infected". Other patients were diagnosed either of the above three status from the combination of multiple tests and their chronological changes.

\section{Statistical analysis}

To evaluate serum gastrin, $\mathrm{PGs}$ and vitamin $\mathrm{B}_{12}$ among AIG stages, $t$-test was indicated. To evaluate endoscopic corpus atrophy, chi-square test was indicated. SPSS (Stats Guild Inc. Chiba, Japan) was used in each analysis and a value of $P<0.05$ was considered significant. 


\section{Results}

We found 29 candidates who met the screening criteria and were taken biopsy specimens during the study period. One patient was excluded from the study because the tissue from the corpus was too small to evaluate atrophy of fundic glands. Of the remaining 28 patients, 22 patients $(78.6 \%, 95 \%$ confidence interval 59.0-91.7) were histopathologically confirmed AIG and studied in the following sections.

Of the 22 AIG patients, 7 were men and 15 were women. Age ranged 43-81 years (mean \pm SD $=65.2 \pm$ 10.8 years). Two patients used vitamin $\mathrm{B}_{12}$-containing agents. There were no patients using proton pump inhibitor (PPI). Currently H. pylori-infected patients were not found in any of the 22 AIG patients. On the other hand, 12 (54.5\%) and 10 patients (45.5\%) were diagnosed as past and not infected, respectively. Four patients (18.2\%) and 18 patients $(81.8 \%)$ were histopathologically classified into florid and end stages of AIG, respectively (Table 1, Fig. 1). No patients were histopathologically classified into early stage of AIG.

In 22 AIG patients, anti-PC antibody ranged from 10x to more than 160x (10x in 2 patients, 20x in 6 patients, $40 \mathrm{x}$ in 5 patients, $80 \mathrm{x}$ in 5 patients, $160 \mathrm{x}$ or greater in 4 patients). One patient was also positive for anti-IF antibody.

Serum gastrin was measured in all 22 AIG patients. In these AIG patients, gastrin ranged from 690 to 5300 (mean $\pm S D=2218.2 \pm 1509.6) \mathrm{pg} / \mathrm{mL}$ and all these values exceeded the normal range (172 $\mathrm{pg} / \mathrm{mL}$ ). Of these 22 patients, 4 and 18 patients were included in the florid and end stages, respectively. Serum gastrin was not statistically different between histopathological stages of AIG (florid stage: mean $\pm \mathrm{SD}=1657.5 \pm 362.5 \mathrm{pg} / \mathrm{mL}$; end stage: $2342.8 \pm 1634.3 \mathrm{pg} / \mathrm{mL} ; P=0.14, t$-test), but those who showed higher serum gastrin than $3000 \mathrm{pg} / \mathrm{mL}$ were all in end stage (Fig. 2).

We analyzed cut-off values for PGs with receiver operating characteristic (ROC) curves. The areas under the curve (AUC) of PG I, PG II and PG I/II ratio were $0.81,0.29$ and 0.98 , respectively (Fig. 3), indicating that PG I and PG I/II ratio were suitable for making cut-off values. The optimal cut-off values for PG I and $P G \mathrm{I} / \mathrm{II}$ ratio were suggested as $14.5 \mathrm{ng} / \mathrm{mL}$ (sensitivity $=0.83$, specificity $=0.85$ ) and 2.1 (sensitivity = 1.00 , specificity $=0.95$ ), respectively. We also evaluated Miki's criteria for PG test in diagnosing AIG (Table 2). Sensitivity and specificity of PG $3+$ were 85 and $100 \%$, respectively. On the other hand, sensitivity and specificity of PG 2+ or $1+$ were 90 and $66.7 \%$, respectively. Among histopathologically confirmed AIG patients, PG I ranged from 2.5 to 71.9 (mean $\pm S D=13.3 \pm 19.5$ ) $\mathrm{ng} / \mathrm{mL}$, PG II from 3.7 to 39.2 (mean \pm $S D=10.9 \pm 8.3) \mathrm{ng} / \mathrm{mL}$ and $P G \mathrm{I} / \mathrm{II}$ ratio from 0.3 to 3.5 (mean $\pm S D=1.0 \pm 0.7$ ). $P G \mathrm{I}, \mathrm{PG} \|$ and $P G \mathrm{I} / \mathrm{II}$ ratio were not statistically different between histopathological stages (Table 3). PG test classification revealed $3+$ in 17 (85\%), 2+ in 1 (5\%), $1+$ in $0(0 \%)$ and negative in 2 patients (10\%) (Fig. 4). Of these 20 patients, 4 and 16 patients were included in florid and end stages, respectively, but the results of PG test were not related to the stage of AIG.

Serum vitamin $B_{12}$ was measured in all 22 patients but 2 of them used vitamin $B_{12}$-containing agents and the values were higher than $400 \mathrm{pg} / \mathrm{mL}$. In the rest of 20 patients, vitamin $B_{12}$ ranged from 50 to 320 
(mean $\pm \mathrm{SD}=137.5 \pm 79.2) \mathrm{pg} / \mathrm{mL}$ (Fig. 5). According to the cut-off value (180 pg/mL), 13 patients (65\%) showed low serum vitamin $B_{12}$. Of the 20 patients, 3 and 17 patients were included in florid and end stages, respectively (Fig. 5). Serum vitamin $B_{12}$ was not statistically different between histopathological stages of AIG (florid stage: mean $\pm \mathrm{SD}=147.3 \pm 37.8 \mathrm{pg} / \mathrm{mL}$; end stage: $135.7 \pm 84.3 \mathrm{pg} / \mathrm{mL} ; P=0.83, t$ test). All 22 patients showed high serum gastrin and 20 of them did not use vitamin $B_{12}$-containing agents. Among them, 7 and 13 patients showed normal and low serum vitamin $B_{12}$, respectively (Fig. 6). On the other hand, in the 20 patients without vitamin $B_{12}$ users, 7 and 13 showed normal and low serum vitamin $B_{12}$, respectively. All the patients with low serum vitamin $B_{12}$ showed high serum gastrin (Fig. 6 ).

Endoscopic corpus atrophy was evaluated in all the 22 AIG patients. Among them, 20 (90.9\%), 1 (4.5\%) and 1 (4.5\%) were classified in 0-p, 0-3 and 0-1, respectively. Therefore, all the 22 AIG patients had endoscopic atrophy 0-1 or greater. Of 4 patients in florid stage, 3 were classified in 0-p and one in 0-3. Of 18 patients in end stage, 17 were classified in 0-p and one in 0-1. There was no significant difference in 0-p ratio between florid and end stage ( $75 \%$ vs $94.4 \%$, respectively, $P=0.22$, chi-square test, Table 4 ).

We counted the number of positive items among the 4 tests: elevated serum gastrin, endoscopic 0-p atrophy, $3+P G$ test and low serum vitamin $B_{12}$. Four items were positive only in 11 AIG patients (50\%). On the other hand, the number of positive items was 1 in 1 patient, 2 in 3 patients and 3 in 7 patients, respectively (Table 1). Of the 7 patients whose number of positive items was 3,6 patients had normal serum vitamin $B_{12}$ and 1 had $2+P G$ test. Common clinical findings in the confirmed AIG patients were $10 x$ or greater anti-PC antibody, elevated serum gastrin greater than $172 \mathrm{pg} / \mathrm{mL}$ and endoscopic atrophy 0-1 or greater. Other items were not common in all the 22 AIG patients.

\section{Discussion}

In the present study, 22 of $28(78.6 \%)$ patients those who met the screening criteria for candidates were histopathologically confirmed as AIG. Our screening criteria worked well with $78.6 \%$ positive predictive value. Currently $H$. pylori-infected patients were not found in any of the 22 AIG patients. We could not find any AIG patients in histopathologically early stage but found 4 in florid and 18 in end stages. We could not find a significant difference between florid and end stages in the items studied. We extracted common clinical findings in the confirmed AIG patients: 10x or greater anti-PC antibody, elevated serum gastrin greater than $172 \mathrm{pg} / \mathrm{mL}$ and endoscopic atrophy 0-1 or greater. We found that PG I and PG I/II ratio may be useful to screen AIG according to ROC analyses, and the optimal cut-off values of PG I and PG I/II ratio were $14.5 \mathrm{ng} / \mathrm{mL}$ and 2.1 , respectively.

We evaluated serum gastrin in AIG patients. In 22 patients who were histopathologically diagnosed AIG, serum gastrin was markedly elevated in both florid and end stages (Table 1). Although serum gastrin was not statistically different between florid and end stages of AIG, those who showed higher serum gastrin than $3000 \mathrm{pg} / \mathrm{mL}$ were all in end stage (Fig. 2). These results suggest that gastrin increases with the progress of histopathological AIG. In addition, the normal cut-off value of serum gastrin $(172 \mathrm{pg} / \mathrm{mL})$ could also be a suitable cut-off value for screening AIG. Because no patients were classified into early 
stage and we studied only a small number of patients in florid stage, more numbers of patients are needed to determine the precise cut-off value to screen AIG.

The present study is the first study to evaluate the ability of PG test to screen candidates of AIG. Using Miki's criteria, 3+ PG test showed high sensitivity and specificity in the present study (Table 2). According to the ROC curves and AUC analyses, PG I and PG I/II ratio can be used for screening AIG (Fig. 3). The optimal cut-off values for PG I and PG I/II ratio were suggested $14.5 \mathrm{ng} / \mathrm{mL}$ and 2.1, respectively. These cut-off values can be proposed as criteria for diagnosing AIG, although the precise cut-off values should be determined with more numbers of patients. We also investigated the differences of PGs between histopathological stages (Table 3). However, the PGs and PG I/II ratio were not statistically different between florid and end stages. In addition, PG test evaluation with Miki's criteria was not related to the histopathological stages (Fig. 4). More numbers of patients may be needed to evaluate PG test in each histopathological stage.

We also included low serum vitamin $B_{12}$ in the screening criteria for AIG. In the present study, 13 patients $(65 \%)$ showed low serum vitamin $B_{12}$, and all the patients with low serum vitamin $B_{12}$ showed high serum gastrin (Fig. 6). On the contrary, not all patients with high serum gastrin showed low serum vitamin $B_{12}$ (Fig. 6). These facts indicate that serum gastrin exceeds the normal range before serum vitamin $B_{12}$ falls. In other words, serum gastrin elevates first and vitamin $B_{12}$ falls later in the progress of AIG. Therefore, low serum vitamin $B_{12}$ may be a marker of severe fundic atrophy in AIG, but we could not obtain clear evidence for the relationship between serum vitamin $B_{12}$ and the histopathological stages in the present study (Fig. 5).

We evaluated endoscopic corpus atrophy in all the 22 AIG patients with Updated Kimura-Takemoto classification [7, 8]. Among them, 21 (95.5\%) were classified in 0-p or 0-3, and one patient was classified in 0-1 atrophy. This fact suggests that AIG is strongly suspected in patients classified in 0-p or 0-3. The common finding of endoscopic atrophy was at least 0-1 atrophy. Because there was not a significant difference in 0-p ratio between florid and end stages, endoscopic corpus atrophy was not always related to the histopathological stages (Table 4). The discrepancy between endoscopic and histopathological atrophy may be due to the difference between macroscopical and microscopical points of view. Endoscopic diagnosis of atrophy is a lateral overall diagnosis including mucosa and deeper layers whereas histopathological diagnosis from biopsy samples is a point diagnosis of the surface part of gastric wall. In addition, histopathological diagnosis is largely affected with the part where biopsy specimen was taken. It is reported that remnant fundic glands exist in some AIG patients [14], so that gastric atrophy may not be diagnosed microscopically if the biopsy sample was taken from the remnant fundic mucosa. These may be the possible reasons why histopathological stages were not related to endoscopic atrophy.

We screened AIG with autoantibodies and four non-invasive tests in the present study. The number of positive items in the latter four tests ranged from 1 to 4 (Table 1). The only common item in the AIG patients examined was elevated serum gastrin. It suggests that elevated serum gastrin may be essential 
to diagnose AIG and may be enough for the screening. The cut-off value of manufacturer's instruction $(172 \mathrm{pg} / \mathrm{mL})$ was suitable in the subjects of this study. Because patients with renal dysfunction or using PPI tend to have elevated serum gastrin $[15,16]$, it is not suitable to use serum gastrin to screen AIG in such patients. In addition, endoscopic findings and PG tests also tend to show abnormal in PPI users [17, 18], so that serum vitamin $B_{12}$ test may be an only trustable test to screen AIG in such patients. However, serum vitamin $B_{12}$ was decreased in only 13 of 20 AIG patients (65\%) (Fig. 5), and those who take vitamin $B_{12}$-containing agents had an increased serum $B_{12}$, it may not be enough to screen AIG with single serum vitamin $B_{12}$ test, but adding vitamin $B_{12}$ test may be recommended.

No patients were histopathologically confirmed as early stage of AIG in the present study. On the other hand, all AIG patients in the present study were either in florid or end stages. Early stage of AIG may be difficult to screen with our criteria. We could not find significant differences between florid and end stages in serum gastrin, $P G s$, vitamin $B_{12}$ or endoscopic atrophy in the present study. These facts may indicate that there is no major clinical difference between florid and end stages and suggest that florid stage is already an advanced stage of AIG in clinical aspects. Our criteria may be able to screen only advanced stages of AIG. We may need to find other criteria to screen early stage of AIG.

The present study had some limitations. First, this was a retrospective observational study performed at a single institution. More numbers of patients are needed to determine the power of the screening criteria and to find suitable cut-off values to screen AIG. Second, we could not test anti-IF antibody in all the patients with negative anti-PC antibody test, because the test was out of insurance coverage in Japan and expensive. Because the sensitivity and specificity of anti-PC antibody are reported to be $81 \%$ and $90 \%$, respectively [19], we may have missed some AIG patients. It is expected that the combination of anti-PC and anti-IF antibody tests increase the diagnostic performance in the future [19].

\section{Conclusions}

Florid stage in histopathology may be already in an advanced stage of AIG in clinical aspects as well as end stage. Our screening criteria without biopsy are applicable to screen clinically advanced AIG with 78.6\% positive predictive value. PG I and PG I/II ratio may be useful to screen AIG, and the optimal cut-off values of PG I and PG I/II ratio were $14.5 \mathrm{ng} / \mathrm{mL}$ and 2.1, respectively. However, we may need other criteria to screen early stage of AIG.

\section{Abbreviations}

AIG

PC

IF

PG
Autoimmune gastritis

Parietal cell

Intrinsic factor

Pepsinogen 
ECL Enterochromaffin cell-like

H. pylori Helicobacter pylori

HpAb H. pylori antibody

HpSA H. pylori stool antigen

UBT Urea breath test

PPI Proton pump inhibitor

ROC Receiver operating characteristic

AUC Areas under the curve

\section{Declarations}

\section{Ethics approval}

We conducted the present study according to the Declaration of Helsinki and the Ethical Guidelines for Medical and Health Research Involving Human Subjects issued from the Japan Ministry of Health, Labour and Welfare in 2015. The present study was approved by the Institutional Review Board of JCHO Shiga Hospital (approval number 2018-16).

\section{Consent for publication}

The present study is a retrospective observational study and the study participants did not give written informed consent. Those who wanted to withdraw from the study were given a chance to opt out of the study as recommended by the Ethical Guidelines for Medical and Health Research Involving Human Subjects issued from the Japan Ministry of Health, Labour and Welfare. Table and figures did not contain any non-anonymous data.

\section{Availability of data and materials}

The datasets during and/or analyzed during the current study available from the corresponding author on reasonable request.

\section{Competing interests}

The authors declare that they have no conflict of interest.

\section{Funding}


This research did not receive any specific grant from any funding agency in the public, commercial or notfor-profit sector.

\section{Authors' contributions}

YW and SN designed the study, made statistical analysis, interpreted the data and wrote the manuscript. $\mathrm{NM}, \mathrm{RC}, \mathrm{MO}, \mathrm{MF}, \mathrm{HH}$ and $\mathrm{KH}$ contributed to screeen candidates and discussed for the manuscript. ST and RK performed histopathological diagnosis and discussed for the manuscript. KM supervised the study and discussed for the mnuscript. All authors approved the final version of the manuscript.

\section{Acknowledgements}

We would greatly appreciate Mr. Shinji Umano and Ms. Akiko Yoshida for preparation of biopsy specimens.

\section{References}

1. Strickland RG, Mackay IR. A reappraisal of the nature and significance of chronic atrophic gastritis. Am J Dig Dis. 1973;18:426-40.

2. Kim J, Kim MJ, Kho HS. Oral manifestations in vitamin $B_{12}$ deficiency patients with or without history of gastrectomy. BMC Oral Health. 2016;16:60.

3. Kawanaka M, Tanikawa T, Kamada T, et al. High prevalence of autoimmune gastritis in patients with nonalcoholic steatohepatitis. Intern Med. 2019;58:2907-13.

4. Notsu T, Adachi K, Mishiro T, et al. Prevalence of autoimmune gastritis in individuals undergoing medical checkups in Japan. Intern Med. 2019;58:1817-23.

5. Cabrera de León A, González DA, Almeida AA, et al. Factors associated with parietal cell autoantibodies in the general population. Immunol Let. 2012;147:63-6.

6. Zhang Y, Weck MN, Schöttker B, et al. Gastric parietal cell antibodies, Helicobacter Pylori infection, and chronic atrophic gastritis: evidence from a large population-based study in Germany. Cancer Epidemiol Biomarkers Prev. 2013;22:821-6.

7. Nakajima $S$, Watanabe $H$, Shimbo T, et al. Incisura angularis belongs to fundic or transitional gland regions in Helicobacter pylori-naïve normal stomach: Sub-analysis of the prospective multi-center study. Dig Endosc. 2021;33:125-32.

8. Kimura K, Takemoto T. An endoscopic recognition of the atrophic border and its significance in chronic gastritis. Endoscopy. 1969;1:87-97.

9. Miki K, Urita Y. Using serum pepsinogens wisely in a clinical practice. J Dig Dis. 2007;8:8-14.

10. Mori N, Nakajima S, Takemura S, et al. Primary diagnosis of autoimmune gastritis without biopsy. Journal of Gastrointestinal Cancer Screening. 2020;58:423-35. (in Japanese).

11. Greenson JK, Lawers GY, Montgomery EA, et al. Diagnostic pathology; Gastrointestinal. 3rd ed. Amsterdam: Elsevier; 2019. pp. 140-3. 
12. Coati I, Fassan M, Farinati F, et al. Autoimmune gastritis: Pathologist's viewpoint. World $\mathrm{J}$ Gastroenterol. 2015;21:12179-89.

13. Wada Y, Nakajima S, Kushima R, et al. Pyloric, pseudopyloric, and spasmolytic polypeptideexpressing metaplasias in autoimmune gastritis: a case series of 22 Japanese patients. Virchows. 2021. doi:10.1007/s00428-021-03033-5.

14. Terao S, Suzuki S, Yaita H, et al. Multicenter study of autoimmune gastritis in Japan: Clinical and endoscopic characteristics. Dig Endosc. 2020;32:364-72.

15. Kes R. Serum gastrin concentration in chronic renal failure. Acta Med Croatica. 1992;46:47-58.

16. Yang YX, Metz DC. Safety of proton pump inhibitor exposure. Gastroenterology. 2010;139:1115-27.

17. Li Z, Wu C, Li L, et al. Effect of long-term proton pump inhibitor administration on gastric mucosal atrophy: A meta-analysis. Saudi J Gastroenterol. 2017;23:222-8.

18. Festen HP, Tuynman HA, Défize J, et al. Effect of single and repeated doses of oral omeprazole on gastric acid and pepsin secretion and fasting serum gastrin and serum pepsinogen I levels. Dig Dis Sci. 1986;31:561-6.

19. Lahner E, Norman GL, Severi C, et al. Reassessment of intrinsic factor and parietal cell autoantibodies in atrophic gastritis with respect to cobalamin deficiency. Am J Gastroenterol. 2009;104:2071-9.

\section{Tables}

Tables 1 to 4 are only available as a download in the Supplemental Files section.

\section{Figures}

(a)

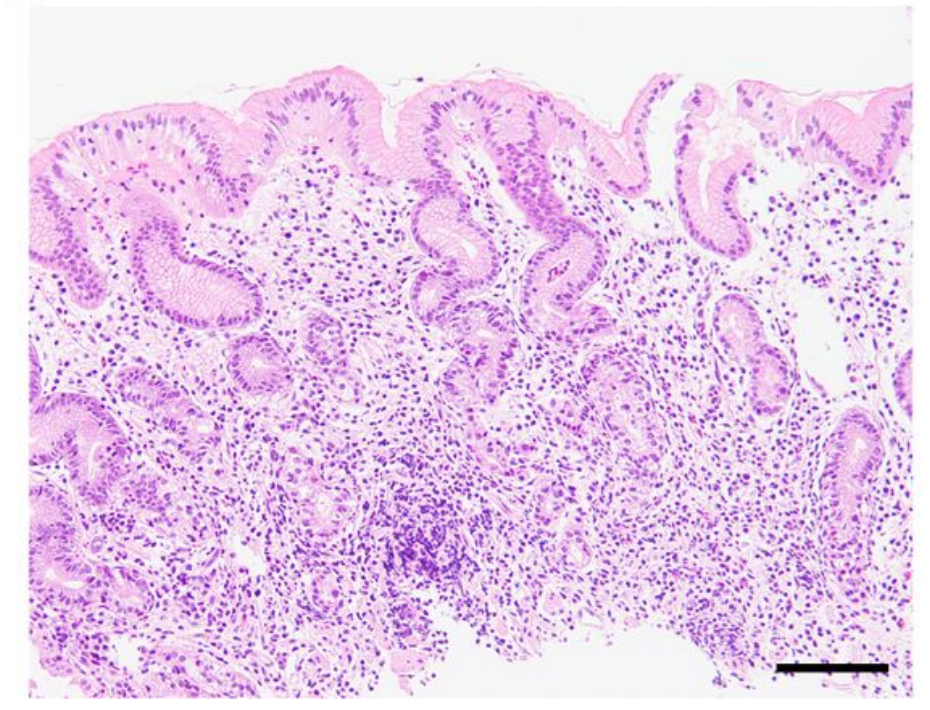

(b)

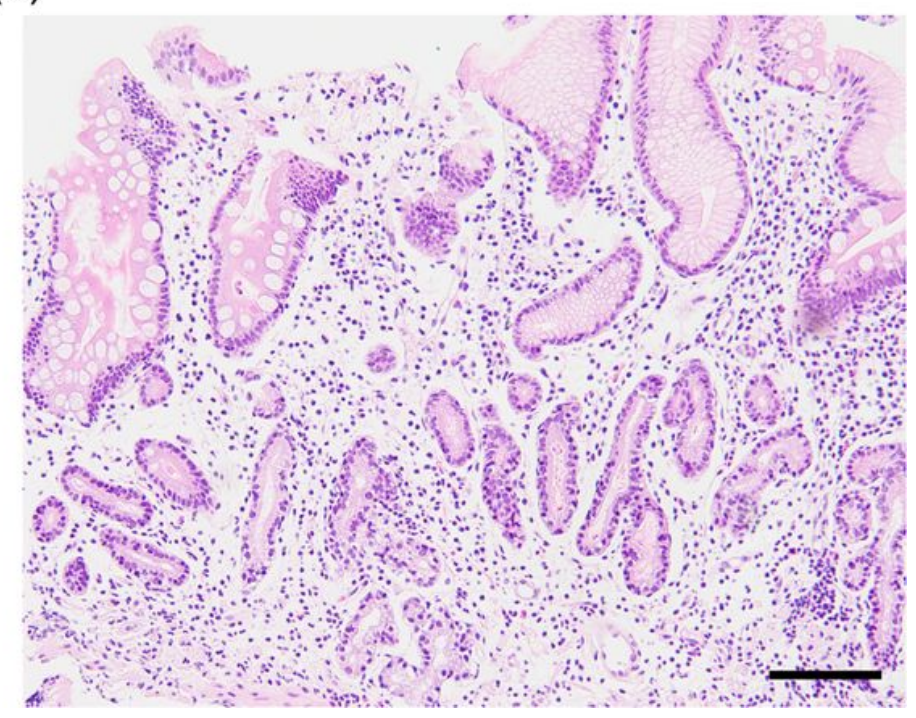

Figure 1 
Light microscopic pictures of florid and end stages (H\&E stain).

The florid stage consisted of marked decrease of fundic glands with lymphoplasmacytic infiltration predominantly in the deeper part of the mucosa (a). The end stage consisted of complete loss of fundic glands with minimal inflammatory cell infiltration (b). Scale bar: $200 \mu \mathrm{m}$.

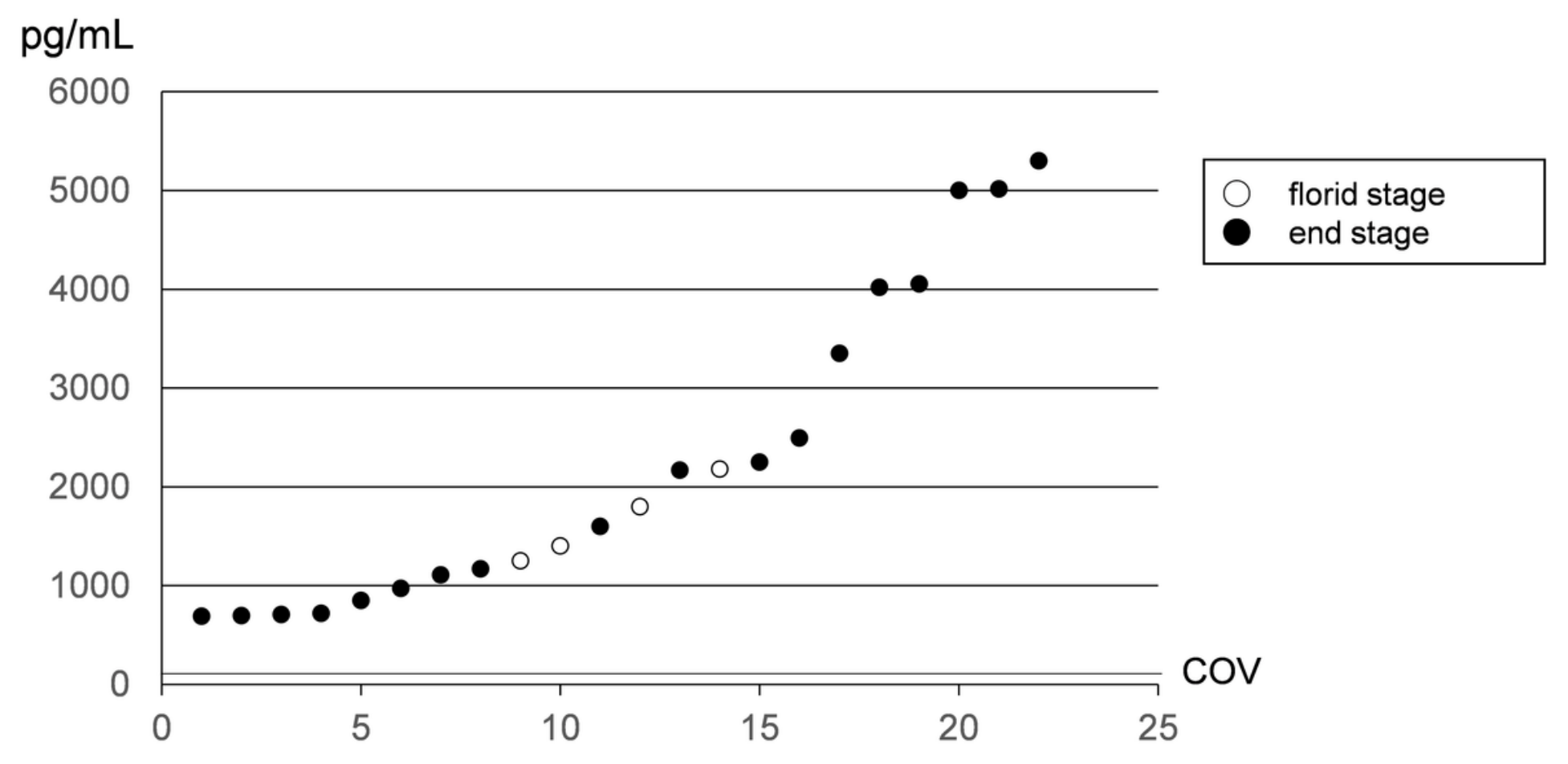

Figure 2

Distribution of serum gastrin of all AIG patients $(\mathrm{N}=22)$.

COV; cut-off value $(172 \mathrm{pg} / \mathrm{mL})$

The numbers in the horizontal axis were not patient's numbers in Table 1. Patients are ordered by the values of serum gastrin. All 22 patients showed high serum gastrin. Four patients were included in florid stage (๑), and 18 patients were included in end stage $(\bullet)$. 
(a)

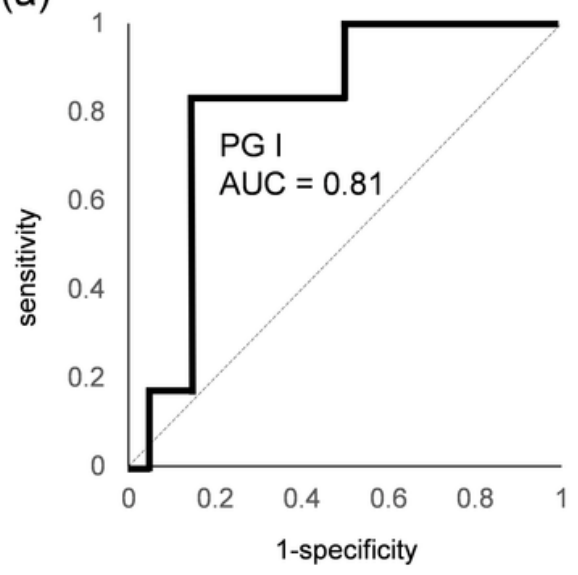

(b)

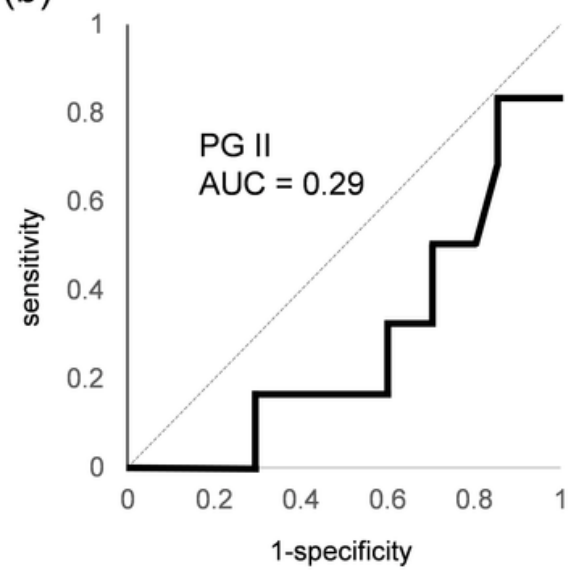

(c)

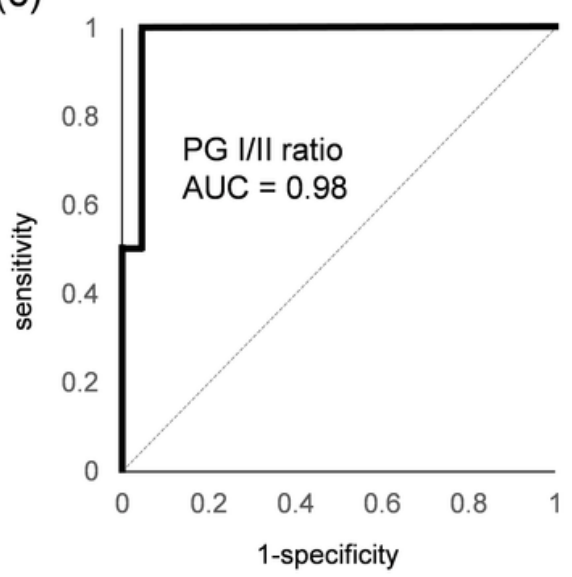

Figure 3

Receiver operating characteristic (ROC) curves of PG I (a), PG II (b) and PG I/II ratio (c).

PG; pepsinogen, AUC; area under the curve

AUC of PG I, PG II and PG I/II ratio were $0.81,0.29$ and 0.98 , respectively.

According to ROC curve analyses, the optimal cut-off values of PG I and PG I/II ratio were $14.5 \mathrm{ng} / \mathrm{mL}$

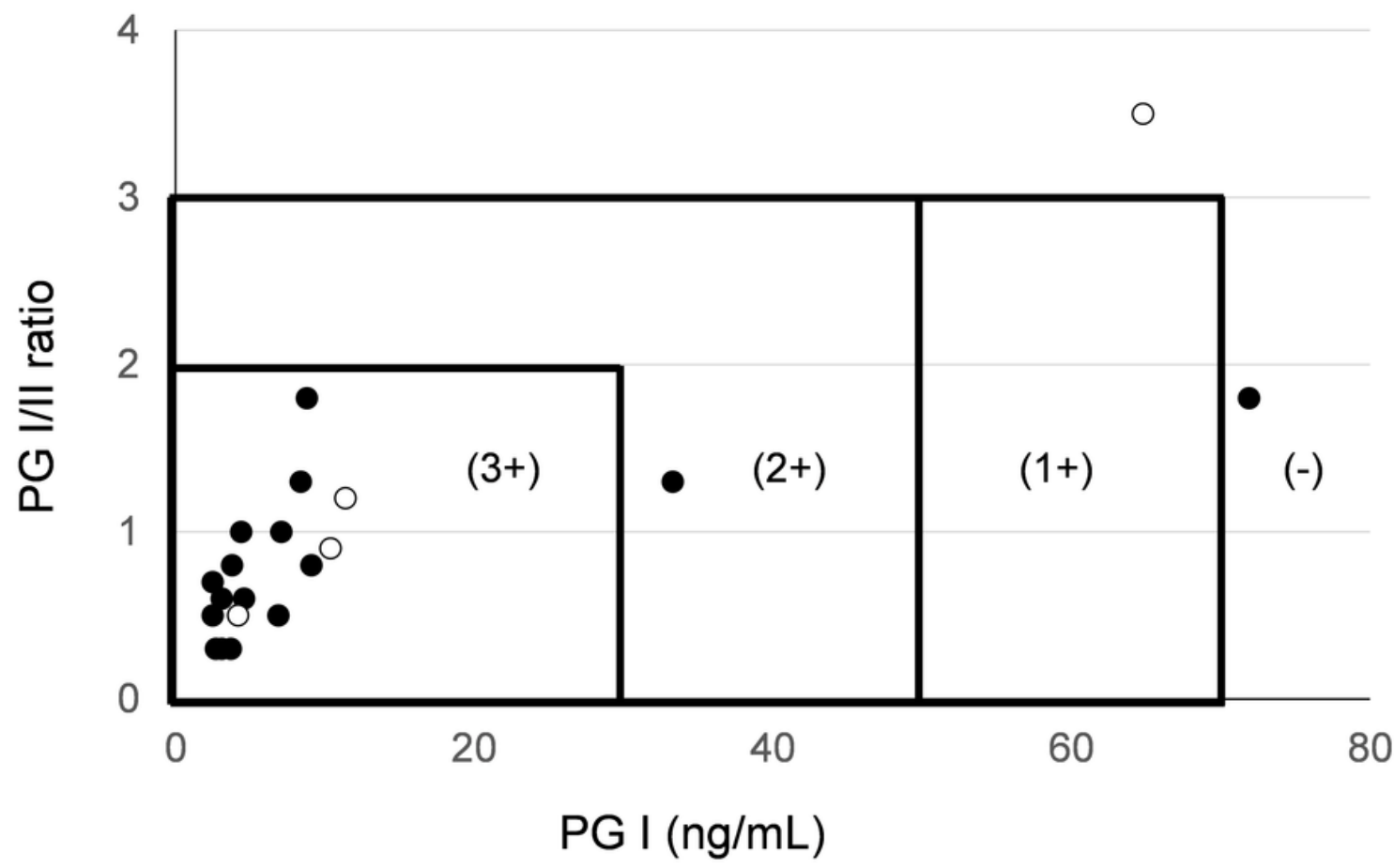

Figure 4

Distribution of PG test of 20 AIG patients $(\mathrm{N}=20)$.

PG; pepsinogen 
PG test classification revealed 3+ in 17 (85\%), 2+ in 1 (5\%), 1+ in $0(0 \%)$ and negative in 2 patients (10\%). Four patients were included in florid stage $(\bullet)$, and 16 patients were included in end stage $(\bullet)$.

\section{$\mathrm{pg} / \mathrm{mL}$}

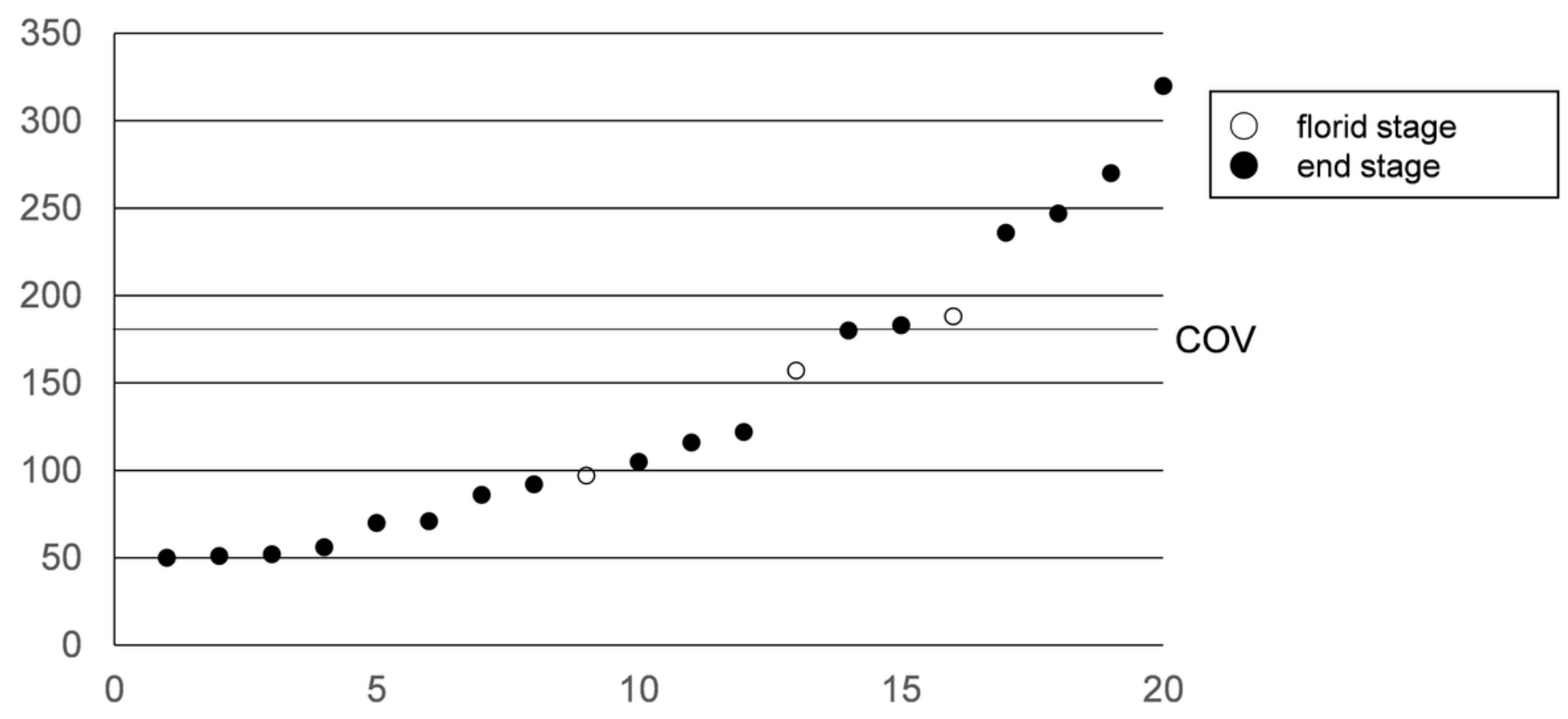

\section{Figure 5}

Distribution of serum vitamin $B_{12}(N=20)$.

COV; cut-off value $(180 \mathrm{pg} / \mathrm{mL})$

Vitamin- $\mathrm{B}_{12}$ users were not included in the figure.

The numbers in the horizontal axis are not the patient's numbers in Table 1. Patients are ordered by the values of serum vitamin $B_{12}$. Thirteen patients $(65 \%)$ showed low serum vitamin $B_{12}$. Three patients were included in florid stage $(\mathbf{\bullet})$, and 17 patients were included in end stage $(\mathbf{\bullet})$. 

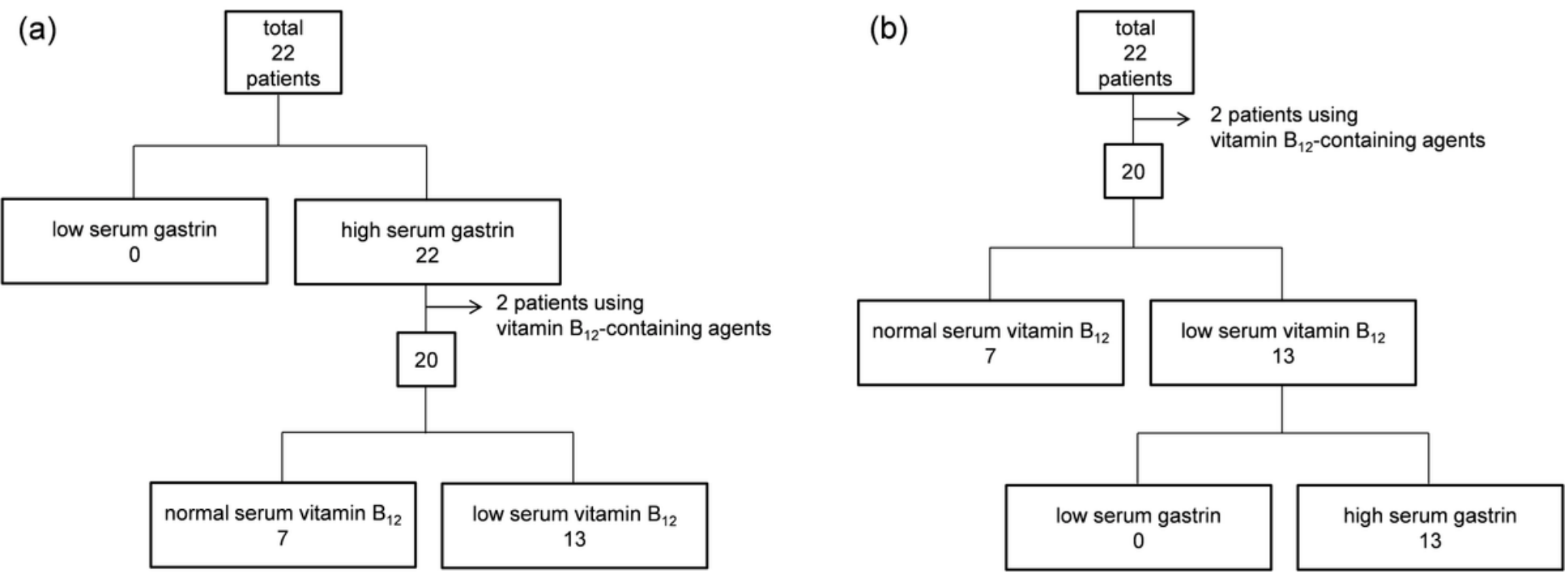

Figure 6

Diagram of AIG patients according to serum gastrin (a) and serum vitamin $B_{12}$ (b).

All the $22 \mathrm{AIG}$ patients showed high serum gastrin. Among them, 20 patients did not use vitamin $\mathrm{B}_{12^{-}}$ containing agents and 13 patients $(65 \%)$ of them showed low serum vitamin $B_{12}$ (a). In the 20 patients who did not use vitamin $B_{12}$-contaning agents, 13 patients showed low serum vitamin $B_{12}$ (b). All of them showed high serum gastrin.

\section{Supplementary Files}

This is a list of supplementary files associated with this preprint. Click to download.

- Table1.xlsx

- Table2.xlsx

- Table3.xlsx

- Table4.xlsx 